\title{
Searches for electroweak SUSY and long-lived particles at the LHC
}

\author{
Hideyuki Oide* on behalf of ATLAS and CMS Collaborations \\ Tokyo Institute of Technology \\ E-mail: oide@phys.titech.ac.jp
}

\begin{abstract}
Each of CMS and ATLAS experiments has collected approximately $140 \mathrm{fb}^{-1}$ of $p p$ collisions at $\sqrt{s}=13 \mathrm{TeV}$ in the LHC Run 2 in the four years of data-taking in 2015-2018. This unprecedented amount of dataset enables to extend the search sensitivity for physics beyond the Standard Model (BSM) especially for targeting relatively low cross-section BSM processes. Pair-production of superpartners of electroweak $(W / Z)$ and higgs bosons in the supersymmetry (SUSY) models, collectively referred to as electroweakinos, or pair-production of superpartners of charged leptons (sleptons) are one of such search areas that can profit from this large amount of dataset in order to gain the sensitivity. The other area of BSM searches, that has been catching more and more attention in recent years is the searches of long-lived particles (LLPs); often LLP searches have zero or small amount of irreducible background events, and search sensitivity is expected to extend with integrated luminosity. This presentation highlights results of the above search areas recently carried out by both CMS and ATLAS experiments.
\end{abstract}

XXVII International Workshop on Deep-Inelastic Scattering and Related Subjects - DIS2019 8-12 April, 2019

Torino, Italy

${ }^{*}$ Speaker. 


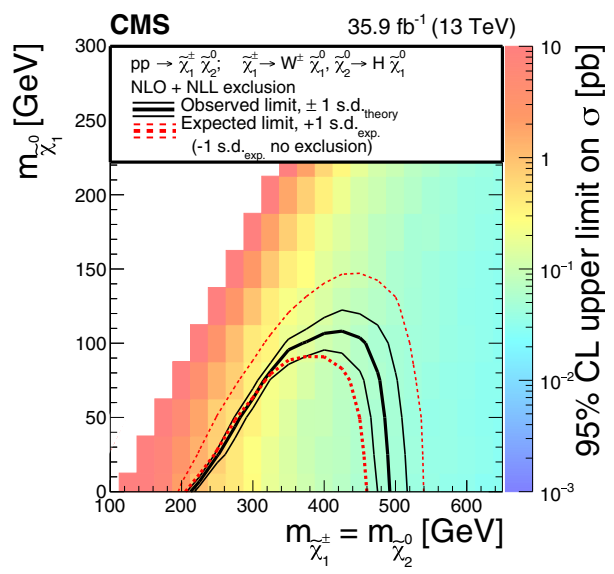

(a)

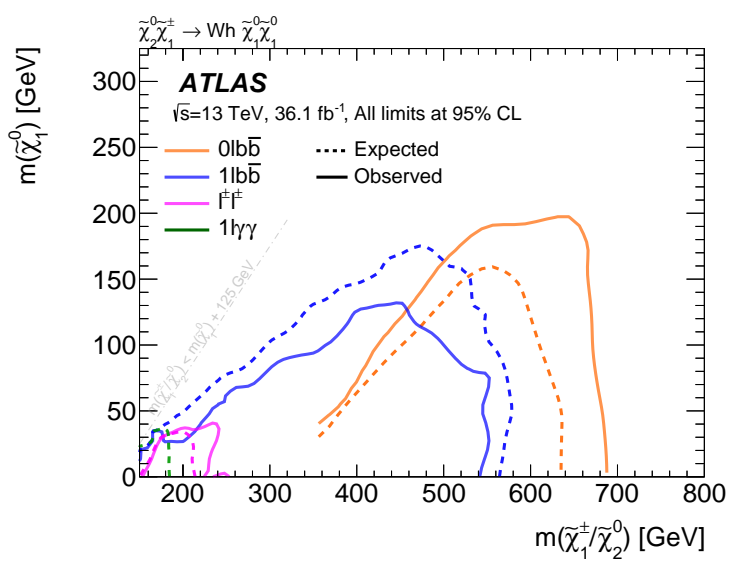

(b)

Figure 1: (a) Excluded limit on $W h \rightarrow 1 \ell+b b$ search for CMS [1] and (b) summary of exclusion of ATLAS Wh searches in the mass plane of $\widetilde{\chi}_{1}^{0}$ and $\widetilde{\chi}_{1}^{ \pm} / \widetilde{\chi}_{2}^{0}[2]$.

\section{Electroweak SUSY searches}

Analogous to the Standard Model (SM) $Z$ boson's case, the mass eigenstates of electroweakinos is represented by a mixture of wino $(\widetilde{W})$, bino $(\widetilde{B})$ and higgsino $(\widetilde{H})$ interaction eigenstates, and they are symbolized by charginos $\widetilde{\chi}_{i}^{ \pm}(i=1,2)$ and neutralinos $\widetilde{\chi}_{i}^{0}(i=1 \ldots 4)$. Typically the lightest neutralino, $\widetilde{\chi}_{1}^{0}$, is assumed to be a stable particle due to the conservation of $R$-parity. In the collider searches, mostly the mass spectrum between $\widetilde{\chi}_{1}^{0}, \widetilde{\chi}_{1}^{ \pm}$and $\widetilde{\chi}_{2}^{0}$ is important, and this configuration depends on the assumed MSSM mass parameters $M_{1}, M_{2}$ and $\mu$. In the LHC, direct pair-productions of lighter electroweakinos, namely $\widetilde{\chi}_{1}^{+} \widetilde{\chi}_{1}^{-}, \widetilde{\chi}_{1}^{ \pm} \widetilde{\chi}_{1}^{0}$ or $\widetilde{\chi}_{1}^{ \pm} \widetilde{\chi}_{2}^{0}$ are mostly studied in the context of SUSY searches ${ }^{1}$. When the mass splitting $\Delta m\left(\widetilde{\chi}_{i}^{ \pm, 0}, \widetilde{\chi}_{1}^{0}\right)$ is greater than SM boson masses of 80-125 GeV, which is typically realized by the "wino-bino" scenario, the decay of $\widetilde{\chi}_{1}^{ \pm}$ emits $W^{ \pm}\left(\widetilde{\chi}_{1}^{ \pm} \rightarrow W^{ \pm} \widetilde{\chi}_{1}^{0}\right)$, and the decay of $\widetilde{\chi}_{2}^{0}$ may emit $Z$ or higgs boson $h\left(\widetilde{\chi}_{2}^{0} \rightarrow Z \widetilde{\chi}_{1}^{0} / h \widetilde{\chi}_{1}^{0}\right)$. Then these processes collectively yield diboson final states. Dedicated searches are needed for the case of compressed spectrum, represented by the "higgsino" search where $\mu$ parameter is supposed to be very light relative to $M_{1}$ and $M_{2}$. Slepton pair production $p p \rightarrow \widetilde{\ell}^{+} \widetilde{\ell}^{-}$is another electroweak SUSY possibility, and this process may share leptonic final states with the electroweakino searches. Searches are typically optimized and interpreted in the scheme of the so-called simplified model that results in maximum possible exclusion. More complex scenarios, e.g. involvement of both electroweakinos and sleptons in the mass spectrum are also studied.

\subsection{Wh searches}

This class of analyses focuses on $\widetilde{\chi}_{1}^{ \pm} \widetilde{\chi}_{2}^{0}$ production where $\widetilde{\chi}_{2}^{0}$ is assumed to emit higgs boson, yielding $W^{ \pm} h$ final states. A number of sub-search channels can be defined by the permutation of $q q$ and $\ell^{ \pm} v$ decay branches of the $W$ boson as well as a variety of the SM-like higgs decays

\footnotetext{
${ }^{1}$ Meanwhile $\widetilde{\chi}_{1}^{0} \widetilde{\chi}_{1}^{0}$ pair production with initial-state radiation of jet, photon or other particles is studied in the context of dark matter searches, collectively referred to as "mono- $X$ " searches.
} 
in $b b, \ell^{ \pm}+X$ or $\gamma \gamma$. The requirement of the higgs boson mass at $125 \mathrm{GeV}$ is the key feature of the search when the higgs invariant mass is reconstructible. Representative search channels are (i) $0 \ell+b b$, (ii) $1 \ell+b b$, (iii) $1 \ell+\gamma \gamma$ and (iv) $2 \ell$ or $3 \ell$ where $\ell=e$ or $\mu$. Due to the branching fraction and the amount of background events, the sensitive regions in the mass parameter space of $\left(m_{\widetilde{\chi}_{1}^{ \pm}} \simeq m_{\widetilde{\chi}_{2}^{0}}, m_{\widetilde{\chi}_{1}^{0}}\right)$ are complementary in these search channels.

For both of the $0 \ell+b b$ and $1 \ell+b b$ cases, the largest SM background contribution comes from the $t \bar{t}$ and $t W$ processes, and requirement of the contransverse mass, $m_{\mathrm{CT}}$, defined as $m_{\mathrm{CT}} \equiv$ $\sqrt{2 p_{\mathrm{T}}^{b_{1}} p_{\mathrm{T}}^{b_{2}}\left(1+\cos \Delta \phi_{b b}\right)}$, to be greater than around $m_{t}$, provides a good rejection of it. While the $0 \ell+b b$ channel is triggered by missing transverse momentum $\left(E_{\mathrm{T}}^{\mathrm{miss}}\right)$ and required having the energetic event (jet) activity by the effective mass $m_{\mathrm{eff}}$, the $1 \ell+b b$ channel is triggered by a single $e$ or $\mu$ and required to have a well-isolated good-quality lepton, and the transverse mass with respect to the lepton, $m_{\mathrm{T}}^{\ell}$, is required to be above around $2 m_{W}$. Both channels are required to have the invariant mass of the two $b$-tagged jets to be consistent with the higgs $125 \mathrm{GeV}$ mass. The CMS result of $1 \ell+b b$ using $36 \mathrm{fb}^{-1}$ of dataset [1], which has two signal regions (SRs) classified by the $E_{\mathrm{T}}^{\text {miss }}$ ranges of $E_{\mathrm{T}}^{\text {miss }} \in[125,200] \mathrm{GeV}$ and $E_{\mathrm{T}}^{\text {miss }}>200 \mathrm{GeV}$, shows the observed event yields consistent with SM, and it excludes $220 \mathrm{GeV}<m_{\widetilde{\chi}_{1}^{ \pm}}<490 \mathrm{GeV}$ for the case of massless $\widetilde{\chi}_{1}^{0}$ (Figure 1(a)). The $0 \ell+b b$ channel is more sensitive to higher mass range than $1 \ell+b b$, partially due to large branching fraction of the signal. The ATLAS result using $36 \mathrm{fb}^{-1}$ of dataset [2] has excluded up to $690 \mathrm{GeV}$ of wino-like chargino $\widetilde{\chi}_{1}^{ \pm}$for massless $\widetilde{\chi}_{1}^{0}$, and this is complementary to the $1 \ell+b b$ channel to cover higher chargino mass region, as shown in Figure 1(b).

The $1 \ell+\gamma \gamma$ channel can offer a clean signal due to the narrow-mass peak requirement of $h \rightarrow$ $\gamma \gamma$, but the SM higgs $V h$ production and other higgs production contribute as the dominant on-peak irreducible background. In the ATLAS search using $36 \mathrm{fb}^{-1}$ of dataset [2], events are triggered by a di-photon trigger with $E_{\mathrm{T}}>35(25) \mathrm{GeV}$ for the leading (sub-leading) photon and required to be $E_{\mathrm{T}}>40(31) \mathrm{GeV}$ in the offline selection. In addition, $b$-jets are vetoed, as well as $E_{\mathrm{T}}^{\text {miss }}>40 \mathrm{GeV}$, $p_{\mathrm{T}}^{\ell}>25 \mathrm{GeV}$, and various transverse masses with respect to (sub-)leading photons and to leptons are required. The di-photon invariant mass is required to be $m_{\gamma \gamma} \in[120,130] \mathrm{GeV}$. Two SRs are defined using the transverse mass with respect to the sub-leading photon, $m_{\mathrm{T}}^{\gamma_{2}}$, and the lepton's transverse mass $m_{\mathrm{T}}^{\ell}$ : (a) $\left(m_{\mathrm{T}}^{\gamma_{2}}>140 \mathrm{GeV}, m_{\mathrm{T}}^{\ell}>110 \mathrm{GeV}\right)$ and (b) $\left(m_{\mathrm{T}}^{\gamma_{2}} \in[80,140] \mathrm{GeV}, m_{\mathrm{T}}^{\ell}<\right.$ $110 \mathrm{GeV}$ ). While the peaking SM higgs contribution is estimated from the NLO MC simulation, the non-peaking continuum background is extracted from side-band of the $m_{\gamma \gamma}$ region in a datadriven manner. This side-band component is estimated to be the dominant background component with larger uncertainty than the irreducible components due to the limited statistics of the side band. Mild upper fluctuation of yields has been observed in both SRs, and the local $p_{0}$-value of the significance is 0.03 and 0.09 respectively. While expected limit of this channel covers up to around $185 \mathrm{GeV}$ chargino mass, the actual observed limit is precluded to draw in this channel due to the mild excess.

\section{2 $W W$ or slepton searches}

Pair production of $\widetilde{\chi}_{1}^{+} \widetilde{\chi}_{1}^{-}$yielding $W^{+} W^{-}$and neutralinos is another electroweak SUSY search branch. When both of $W$ bosons decay leptonically, the process has a signature of dilepton and $E_{\mathrm{T}}^{\mathrm{miss}}$. If the lepton flavor is identical, i.e. $e^{+} e^{-}$or $\mu^{+} \mu^{-}$final states, the signature is common to 
slepton pair-production of $p p \rightarrow \widetilde{\ell}^{+} \widetilde{\ell}^{-} \rightarrow\left(\ell^{+} \widetilde{\chi}_{1}^{0}\right)\left(\ell^{-} \widetilde{\chi}_{1}^{0}\right)(\ell=e, \mu)$ and these scenarios are searched together with slightly different search optimization. The major SM background processes are diboson $(W W, W Z, Z Z)$ and $t \bar{t}$ processes. In the ATLAS search using the full Run 2 dataset of $139 \mathrm{fb}^{-1}$ [3], events are required from opposite-sign dilepton triggers $\left(e^{+} e^{-}, e^{+} \mu^{-}\right.$or $\left.\mu^{+} \mu^{-}\right)$with both identified leptons to satisfy at least $25 \mathrm{GeV}$ in the offline reconstruction. The dilepton invariant mass is required to be greater than $25 \mathrm{GeV}$, and the $Z$ boson veto is applied for same-flavor dilepton channels in the range $m_{Z} \pm 30 \mathrm{GeV}$. Up to one jet is allowed to be present in the event unless it is $b$-tagged, and events are classified by the presence of the jet. In addition $E_{\mathrm{T}}^{\mathrm{miss}}>110 \mathrm{GeV}$ and $E_{\mathrm{T}}^{\text {miss }}$ significance greater than 10 are required. With the above signal selection, events are classified by the stransverse mass $m_{\mathrm{T} 2}[4]$ and $m_{\mathrm{T} 2}>100 \mathrm{GeV}$ is required for the signal. For massless $\widetilde{\chi}_{1}^{0}$, masses up to $420 \mathrm{GeV}$ are excluded for the production of the lightest chargino pairs assuming $W$ boson mediated decays, whereas for slepton-pair production masses up to $700 \mathrm{GeV}$ are excluded assuming three generations of mass-degenerate sleptons.

\section{LLP searches}

\subsection{Direct charged LLP searches}

Massive charged LLPs could be possibly present in various BSM scenarios. One typical example is gluino $R$-hadron production in case of the split-SUSY scenario [5] or pair-production of long-lived sleptons yielding charged leptons and gravitinos in the GMSB scenario [6]. Massive charged LLPs could be directly detected by collider detectors if the flight length is sufficiently long to reach the tracking or outer detectors. Several observables can be used in a complementary manner: (i) short track, (ii) anomalously large ionization loss $(\mathrm{d} E / \mathrm{d} x)$ using charge-sensitive detector and (iii) anomalously large time-of-flight (ToF) using timing-sensitive detectors. The latter two options make use of the fact that massive particles tend to be significantly slower than SM charged stable particles. Combinations of these observables cover broad range of lifetime spanning $\mathscr{O}(0.1 \mathrm{~ns})$ up to $\gtrsim 50 \mathrm{~ns}$ in case where charged LLPs are stable over the detector size.

A recent ATLAS search using $36 \mathrm{fb}^{-1}$ of dataset [7] targets the detector-stable case using both of $\mathrm{d} E / \mathrm{d} x$ measurement in the pixel detector of the inner tracker as well as ToF measurement in both of hadronic tile calorimeter and muon spectrometers. The charged long-lived BSM particle can be reconstructed as if it were a high-momentum "slow" muon. While the $\beta$-value of the particle is estimated from ToF, the $\gamma \beta$-value is estimated from pixel $\mathrm{d} E / \mathrm{d} x$ value. The mass of the particle is then individually estimated by $(p, \beta)_{\mathrm{ToF}}$ and by $(p, \gamma \beta)_{\mathrm{d} E / \mathrm{d} x}$. Depending on particle hypothesis, several strategies are defined to set SRs. The background yield is predicted in a data-driven manner assuming negligible correlation between momentum and ToF or $\mathrm{d} E / \mathrm{d} x$. For the $R$-hadron scenario, two SRs are defined and the observed yields are consistent with the data-driven background estimation. Limits are set to gluino $R$-hadron scenario up to the gluino mass of $2 \mathrm{TeV}$.

\subsection{Displaced decay searches}

The decay products of the LLP could be captured as a distinctive signature largely displaced from the primary $p p$ interaction point, agnostic to the charge of the LLP. A recent CMS search using $36 \mathrm{fb}^{-1}$ of dataset [8] targets on long-lived gluino forming an $R$-hadron which decays hadronically 


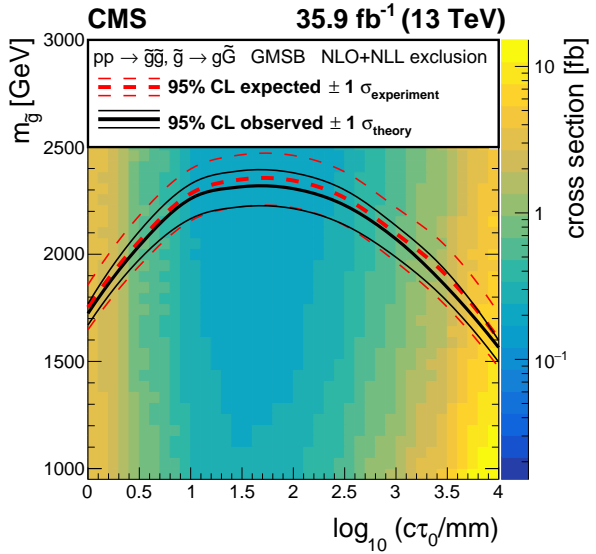

(a)

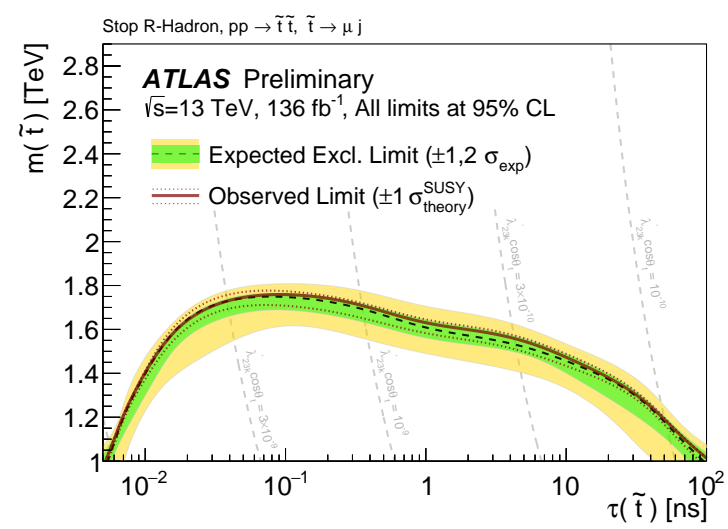

(b)

Figure 2: (a) The limit to $\widetilde{g} \rightarrow g+\widetilde{G}$ in the plane of the gluino mass and its lifetime [8], and (b) the limit to long-lived $R$-parity-violating decay of $\widetilde{t} \rightarrow j+\mu$ in the plane of the gluino mass and its lifetime [9].

by $\widetilde{g} \rightarrow g+\widetilde{G}$ (GMSB) or by $\widetilde{g} \rightarrow t b s$ ( $R$-parity-violation) within the tracking volume. Events are required to pass a dedicated high-level trigger satisfying $H_{\mathrm{T}}>350 \mathrm{GeV}$ and at least two jets with less than two associated prompt tracks and at least one displaced track with $p_{\mathrm{T}}>40 \mathrm{GeV}$, and on top of that $H_{\mathrm{T}}>400 \mathrm{GeV}$ in the offline selection. The jet-seeded displaced vertex (DV) is then reconstructed where seed tracks are selected in the vicinity of the di-jet pairs in the event satisfying a large impact parameter criterion. In order to characterize the degree of displacement, a combined likelihood discriminant is formed using three different features of track multiplicity, displacement significance and track collimation, and a high score threshold is applied to the signal selection. The signal events are classified by $H_{\mathrm{T}}$ and the number of di-jet pairs. The observed events in the SRs are all consistent with the data-driven background estimation, and the limits are set on the models producing displaced jets. Figure 2(a) shows the case for the GMSB $\widetilde{g} \rightarrow g+\widetilde{G}$ scenario scanned over various lifetime, and the gluino mass up to $2.4 \mathrm{TeV}$ is excluded around the lifetime $\tau \sim 0.1 \mathrm{~ns}$ with the maximum sensitivity.

Another type of displaced signature search performed by ATLAS using the full Run 2 dataset [9] targets at the presence of a DV and a non-prompt muon in the event. A BSM scenario yielding this signature is pair-production of top squarks, where each of those decays to a jet and a muon via an $R$-parity-violating coupling, $\widetilde{t} \rightarrow j+\mu$. In order to reconstruct DVs, events are triggered by either $E_{\mathrm{T}}^{\text {miss }}$ or a displaced muon trigger, and they are reserved in a dedicated data stream for a special reconstruction which enables track reconstruction with large impact parameters. DVs are inclusively reconstructed from tracks satisfying $\left|d_{0}\right|>2 \mathrm{~mm}$ and $p_{\mathrm{T}}>1 \mathrm{GeV}$. Compared to the previous reconstruction algorithm used in the $2015+2016$ dataset in the DVs with $E_{\mathrm{T}}^{\text {miss }}$ search [10], the reconstruction is improved to accept more tracks to be associated to the vertex, resulting in enrichment of the representative vertex properties of track multiplicity $n_{\text {track }}$ and visible invariant mass $m_{\mathrm{vis}}$ that are used for signal selection [11]. In the signal selection, two mutually exclusive signal regions are allocated respecting $E_{\mathrm{T}}^{\mathrm{miss}}$ and muon trigger. Events are required to have a muon with 
$p_{\mathrm{T}}>25 \mathrm{GeV}$ and $\left|d_{0}\right|>2 \mathrm{~mm}$, and a DV satisfying $n_{\text {track }} \geq 3$ and $m_{\text {vis }}>20 \mathrm{GeV}$ where the selected muon is allowed to be a constituent track of the DV. The background is classified into main three sources, cosmic, heavy-flavor and fake, and for each of the sources a data-driven yield estimation is performed. The observed events are consistent with the background estimation, and the limit for $R$-parity-violating $\widetilde{t} \rightarrow j+\mu$ process is shown in Figure 2(b). The sensitivity is maximized around lifetime of $\tau \sim 0.1 \mathrm{~ns}$ where the top squark mass up to $1.7 \mathrm{TeV}$ is excluded. Although the long-lived top squark is considered in the scenario, this mass limit is even higher than the prompt top squark searches, illustrating the merit of having quite low-background environment by the requirement of displacement.

\section{Summary}

Intensive searches have been carried out for relatively low cross-section SUSY scenarios on electroweak processes as well as long-lived particle cases in the Run 2 of the LHC using unconventional search techniques. A few very early results using the full dataset of Run 2 were also shown in this presentation. Despite no observed SUSY or BSM evidence have been observed so far, more of searches are ongoing to exploit the LHC Run 2 data-taking which may reveal the presence of new physics.

\section{References}

[1] CMS Collaboration, JHEP 11 (2017) 029, arXiv:1706.09933

[2] ATLAS Collaboration, arXiv:1812.09432, submitted to PRD

[3] ATLAS Collaboration, ATLAS-CONF-2019-008

[4] A.Barr, C. Lester, P. Stephens, Phys.G29:2343-2363,2003

[5] N. Arkani-Hamed et al., arXiv:1212.6971

[6] G. F. Giudice and R. Rattazzi, Phys. Rept. 322 (1999) 419, arXiv:hep-ph/9801271

[7] ATLAS Collaboration, PRD 99 (2019) 092007, arXiv:1902.01636

[8] CMS Collaboration, PRD 99 (2019) 032011, arXiv:1811.07991

[9] ATLAS Collaboration, ATLAS-CONF-2019-006

[10] ATLAS Collaboration, PRD 97 (2018) 052012, arXiv:1710.04901

[11] ATLAS Collaboration, ATLAS-PHYS-PUB-2019-019 